Cortex, Special Issue "Confabulation and Related Disorders"

CORTEX-D-16-00283R1: $2^{\text {nd }}$ Revision, submitted to on 17 Oct 2016

Special guest editors: GF Dalla Barba, MD Kopelman

\title{
What does extinction have to do with confabulation?
}

\author{
Armin Schnider, MD ${ }^{1,2}$; Louis Nahum, PhD ${ }^{1,2}$; Radek Ptak, PhD ${ }^{1,2}$
}

Laboratory of Cognitive Neurorehabilitation, Division of Neurorehabilitation, University of Geneva, CH-1205 Geneva, Switzerland

Division of Neurorehabilitation, Department of Clinical Neurosciences, University Hospital of Geneva, CH-1205 Geneva, Switzerland

Running title: Extinction and confabulation

\section{Correspondance:}

Prof. Armin Schnider

Service de Neurorééducation

Hôpitaux Universitaires de Genève

Av. de Beau-Séjour 26

CH-1206 Geneva / Switzerland

Tel.: +41-22-372 3700; Fax: +41-22-372 3705

e-mail: armin.schnider@hcuge.ch 


\section{Abstract}

Behaviourally spontaneous confabulation denotes a distinct syndrome consisting of confabulations that patients act upon, disorientation, and amnesia. It corresponds to the stable form of the original Korsakoff syndrome. While the syndrome may also occur in confusional states and degenerative dementia, this article is about the syndrome as it occurs after acute and focal brain damage. The patients act according to ideas and obligations that can mostly be traced back to real experiences in their past, but which are not currently valid guides of thinking and behaviour. This inability to abandon behavioural guides (anticipations) that are currently not valid corresponds to a failure of behavioural extinction and to the inability to abandon a previously rewarded choice in reversal learning when the expected reward (outcome) fails to occur, that is, following extinction trials. This article describes evidence from human and animal experiments showing that the posterior medial orbitofrontal cortex, which is typically damaged in these patients, and connected structures of the reward system contain the neural apparatus to signal the non-occurrence of anticipated outcomes, thereby presumably synchronizing thought and behaviour with current reality. Failure of this function, which we call orbitofrontal reality filtering, is associated with behaviourally spontaneous confabulation and disorientation after acute and focal brain damage, but not with other forms of confabulation, and not with reality confusion in degenerative dementia. Potential links with psychosis and decision making will be discussed.

Keywords: Korsakoff syndrome, confabulation, schizophrenia, orbitofrontal cortex, behavioural extinction, decision making 


\section{Introduction}

The term confabulation was proposed by Wernicke (1900) to denote "the emergence of memories of events and experiences which never took place". It replaced Korsakoff's earlier term "pseudo-reminiscences", defined as the "situation in which a patient conceives of an event that he has not really experienced, but that has only come to his mind, as if it had really happened to him" (Korsakoff, 1891). Confabulations were an element of the original Korsakoff syndrome, as defined by Bonhoeffer (1901), which encompassed severe anterograde amnesia, temporally limited retrograde amnesia, disorientation and "a strong tendency to confabulation".

Bonhoeffer (1901) already distinguished two forms of confabulation: (1) Momentary or "out-of-embarrassment" confabulations produced to hide a gap in memory, and (2) fantastic confabulations that go beyond the need to fill a gap in memory. More detailed classifications have been proposed (Moll, 1915). Based on our own observations, Schnider (2008) has proposed to distinguish four forms of memory-related confabulations, which partially or fully dissociate from each other in terms of mechanism and anatomy (Nahum, Bouzerda-Wahlen, Guggisberg, Ptak, \& Schnider, 2012; Schnider, 2008):

(1) Intrusions in memory tests (simple provoked confabulations). These are independent from other confabulations and disorientation and tend to correlate with correctly retrieved items. They seem to be the price for retrieval of more information than is actually available in memory.

(2) Momentary confabulations, that is, false verbal statements that patients make in response to questions or in a situation provoking a comment. These confabulations can be explored with questionnaires. There is no definite mechanism or anatomical basis. They may be associated with impaired mental 
flexibility, a tendency to fill gaps in memory, or they may be part of the following form of confabulation (Nahum et al., 2012).

(3) Behaviourally spontaneous confabulations, which occur in the context of amnesia and reflect a confusion of reality: patients are disoriented when asked about present circumstances (time, location, current duties etc.) and at least intermittently act according to their confabulations. This syndrome corresponds to the stable form of the originally described Korsakoff syndrome (Bonhoeffer, 1901), that is, when it occurs in patients who are no longer in a confusional state. This article is about this form of confabulation as it occurs after acute or focal brain damage.

(4) Fantastic confabulations, which are illogical, often non-sensical and incoherent ideas as they can be observed in confusional states, decompensated psychosis and advanced dementia.

What would reality confusion, as evident from confabulations that patients act upon and disorientation, have to do with behavioural extinction (called extinction in the following)? Extinction denotes a learning process in which the repeated non-occurrence of an anticipated outcome (e.g., a reward) leads to the gradual abandonment of the anticipation and the associated behaviour (Pavlov, 1927). Reward denotes a situation or a stimulus, which reinforces behaviour (Rolls, 1999). The reward system is a loosely defined brain system that processes the type and the occurrence (or non-occurrence) of reinforcing outcomes. While reward processing is often portrayed as quasi-equivalent with pleasure and emotion (Grabenhorst \& Rolls, 2011), we will present evidence that the "reward system" also processes outcomes that have no tangible pleasure (hedonic) value. Core anatomical structures of the system are the orbitofrontal cortex, subcortical dopaminergic structures (substantia nigra, striatum), and the amygdala (Grabenhorst \& 
Rolls, 2011). Of particular interest here will be the orbitofrontal cortex (OFC), the dopaminergic structures, and their connections. This system not only processes the occurrence of rewards (outcomes), but also their non-occurrence (Schultz, Dayan, \& Montague, 1997). Of particular interest for this paper will be the neural activities, which signal the non-occurrence of expected outcomes. We will use the term extinction capacity for the ability to integrate the non-occurrence of anticipated outcomes at specific points in time into the concept of current reality and to adapt behaviour accordingly. Thus, the term is used here to describe the signalling of singular events as it is necessary for rapid behavioural adaptation in reversal learning, rather than the learning process by repeated exposure which characterizes full behavioural extinction. We will provide evidence that the capacity to use this signal is necessary for humans to synchronize their thinking and behaviour with current reality ("the now") and that its failure induces reality confusion with confabulation and disorientation.

\section{The clinical syndrome of behaviourally spontaneous confabulation}

In most cases, behaviourally spontaneous confabulation firstly manifests as confabulations in discussions. Patients recount recent events and experiences as well as plans for the day, which do not correspond to reality and which may appear completely invented. Very often, however, these ideas can be traced back to real events and activities in the patient's past (Korsakoff, 1891; Schnider, von Däniken, \& Gutbrod, 1996a). Most notably, and in distinction from "simple" momentary confabulations (form 2 , above), the patients also intermittently act according to these confabulations, as if their ideas related to the current situation. Some examples may clarify the picture:

A 58-year old woman had suffered damage to the right posterior medial OFC following rupture of an aneurysm of the anterior communicating artery. While she did not confabulate at all in memory test (no intrusions) she profoundly misinterpreted her 
current situation. She stood up during a therapy session indicating that she had to feed

her baby, who was over 30 years old at the time, and then desperately searched for him (Schnider, von Däniken, et al., 1996a).

False ideas may be stable for a long period. A 63-year old psychiatrist, who had suffered primarily right posterior medial orbitofrontal damage after rupture of an aneurysm of the anterior communicating artery, was convinced for months that she was actually a staff psychiatrist in our unit. She would leave therapy sessions in the conviction that she had to see patients. She maintained this idea for several months, in addition to a second idea, namely, that she had to organize a reception in the evening. She slapped her husband when she found the fridge empty, convinced that he had hidden the food she had already bought for tonight's reception. She had indeed worked as a psychiatrist until an early retirement 15 years ago and had often arranged big receptions as the wife of a state official until 20 years ago (Schnider, 2008; Schnider, Bonvallat, Emond, \& Leemann, 2005).

Similar long-standing mono-thematic confabulations were observed in a 30-year old female lawyer hospitalized because of limbic encephalitis with very severe amnesia. Damage involved both medial temporal lobes with extension into the posterior medial orbitofrontal area and the striatum. For months, she was convinced that she was currently working as a lawyer and often desperately searched for her files and her colleagues to prepare a session at court. This conviction held for months (Nahum, Ptak, Leemann, Lalive, \& Schnider, 2010).

These patients' confabulations go beyond simple verbal statements; they represent their concept of reality. While the intensity of confabulations may vary -at times being "spontaneous", at times only appearing in discussions- all patients that we have studied with this disorder shared some additional characteristics (Schnider, 2008): 
- They were severely disoriented regarding time, place, and their current situation and obligations. As patients entered our studies only when they were out of a confusional state, orientation to person was intact (Bouzerda-Wahlen, Nahum, Ptak, \& Schnider, 2013; Nahum, Ptak, Leemann, \& Schnider, 2009; Schnider, von Däniken, \& Gutbrod, 1996b). At the stage of entering our studies, they participated in discussions, understood test instructions and collaborated in behavioural testing.

- They were severely amnesic in terms of a bad free recall in verbal memory tests. Recognition memory was often also affected, as in these three patients, but was normal in some patients. Recognition memory did not distinguish between confabulating and non-confabulating amnesic patients and was not predictive of disorientation (Nahum et al., 2012; Schnider \& Ptak, 1999; Schnider, von Däniken, et al., 1996a, 1996b).

- If brain damage was localizable, lesions concerned of the posterior medial orbitofrontal cortex (OFC) or of a brain area directly connected with it (Schnider, 2003,2008 ), such as, the genu of the internal capsule (Schnider, Gutbrod, Hess, \& Schroth, 1996) or the anterior hypothalamus (Ptak et al., 2001). In contrast, patients who "only" had amnesia, with no reality confusion, had the main lesion overlap in the medial temporal lobe, although they might also have lesions of the dorsolateral prefrontal or other neocortical areas (Schnider \& Ptak, 1999; Schnider, von Däniken, et al., 1996b).

\section{Confusing the "now"}

From the early observations on, confabulations elicited theories about their generation, some of which have re-appeared under different headings in the modern literature. Diverse models are explained in detail in other articles in this issue of Cortex. Only very few theories specifically refer to behaviourally spontaneous confabulation, and none 
explicitly refers to reality confusion and disorientation. We will, therefore, first describe our observations and interpretation and then come back to other theories of confabulation, which resemble our model.

A common feature of patients with behaviourally spontaneous confabulation is that their confabulations can often be traced back to real events in their past (Korsakoff, 1891; Schnider, von Däniken, et al., 1996a). The patients mostly enact earlier habits; they do not normally invent new activities. Thus, they mistake memories (thoughts) that do not relate to the "now" (the ongoing reality) as if they did. We tried to measure this failure by having patients make two runs of a continuous recognition task, both composed of the same pictures (Schnider, 2003). The result was the same in several studies: even when the runs were separated by up to an hour, behaviourally spontaneous confabulators, in comparison to similarly amnesic patients and healthy controls, had a marked performance drop in the second and later runs which was due to a relative increase of false positives. All too often, the patients felt that they had just seen a picture within the ongoing run (the "ongoing reality") when indeed they had seen it in a previous run (Nahum et al., 2012; Schnider \& Ptak, 1999; Schnider, von Däniken, et al., 1996a).

Using variants of this experimental approach in patients and healthy subjects, we obtained a detailed picture of the requirements to maintain the sense of reality, a capacity in which patients with behaviourally spontaneous confabulation fail. Our data, detailed in the respective articles, can be summarized as follows:

- The increase of false positives from the second run on correlated very precisely with the degree of disorientation (Bouzerda-Wahlen et al., 2013; Nahum et al., 2012; Schnider, von Däniken, et al., 1996b). 
- Recovery from behaviourally spontaneous confabulations was associated with the recovered ability to control false positives, that is, to maintain performance in the second and later runs (Schnider, Ptak, von Däniken, \& Remonda, 2000).

- Healthy subjects performing different versions of the task activated posterior medial orbitofrontal cortex, area 13, from the second run on (Schnider, Treyer, \& Buck, 2000; Treyer, Buck, \& Schnider, 2006). In a task using various types of stimuli, this activity extended to the striatum, substantia nigra and medial thalamus (Treyer, Buck, \& Schnider, 2003). These structures are part of the reward system, but also of frontal subcortical loops that connect specific areas of the prefrontal cortex through the basal ganglia, substantia nigra and the thalamus to themselves (Alexander, DeLong, \& Strick, 1986).

- Using high-density elecroencephalography (EEG), we found that the treatment of the stimuli, on which reality confusing patient failed, occurs at 200-300 milliseconds after stimulus presentation, before the brain distinguishes between new and repeated items at 400-600 ms (Schnider, Valenza, Morand, \& Michel, 2002; Wahlen, Nahum, Gabriel, \& Schnider, 2011). In other words, the brain determines whether a memory relates to present reality or not at an early stage of processing, at 200-300 milliseconds, before the content of the upcoming memory (thought) is recognized. The cortical consequence of the orbitofrontal signal appears to be transient inhibition of extended neocortical synchronization when an upcoming thought does not relate to present reality (Bouzerda-Wahlen, Nahum, Liverani, Guggisberg, \& Schnider, 2015; Schnider, 2003).

- As suggested by the evoked potential studies, the orbitofrontal signal indicating that an upcoming memory does not relate to the present, appears to be a phasic, brief signal. Accordingly, inhibition of the orbitofrontal cortex by transcranial direct 
current stimulation (tDCS) (Manuel, David, Bikson, \& Schnider, 2014) and

pharmacological manipulation of dopaminergic transmission (Schnider, Guggisberg, Nahum, Gabriel, \& Morand, 2010), both of which have a long-acting effect, succeeded only in worsening performance in a rapid version of the task, but no manipulation succeeded in improving performance in healthy subjects.

These observations indicate that the memory process, on which behaviourally spontaneous confabulators fail, is an early one (200-300 ms), conveyed by posterior medial orbitofrontal area 13 and apparently relayed by subcortical connections to associative neocortex. We have called this capacity "orbitofrontal reality filtering" (Schnider, 2013).

But what is its physiological basis?

\section{Why extinction?}

The failure of behaviourally spontaneous confabulators in repeated runs of a continuous recognition task has proved to be a reliable marker of reality confusion and has opened ways to explore a thought control mechanism necessary to adapt thinking to ongoing reality. It has allowed us to explore the anatomy, the timing, and the pharmacology of orbitofrontal reality filtering. However, performance in the continuous recognition task is only a marker of that thought control mechanism. It does not reveal the mechanism underlying orbitofrontal reality filtering. What is the mechanism whose failure induces reality confusion provoking disorientation and confabulations?

A central characteristic of patients with full-blown behaviourally spontaneous confabulation is their inability to adapt behaviour to current reality. In the examples described above, the mother insisted on the idea of being at home although no element in the environment ever corresponded to this idea. The psychiatrist continued to believe 
that she was a collaborator of our clinic although she would never find a patient or anybody asking for her advice; and she would continue to believe that she had to prepare a reception although nothing confirmed her imagined preparations. The lawyer continued to be convinced that she had to prepare a court session although she would never find the expected files or colleagues.

These patients fail to integrate the absence of anticipated events into their concept of current reality. They continue to act on the basis of ideas (memories) that are currently not valid. In animal experimentation, this failure corresponds to the absence of a reaction to the signal, which indicates the need to abandon a previously rewarded in favour of an alternate behaviour, as in reversal learning, or which, if repeated, would induce behavioural extinction, defined as the ability to learn from the non-occurrence of expected outcomes (rewards) (Pavlov, 1927). This concept requires one to accept two prerequisites:

1. The principles of outcome-checking not only apply to conditioning and related forms of learning -as used in animal experimentation- but also to more flexible forms of explicit memory and thinking;

2. The brain checks outcomes also if they have no tangible hedonic value.

If one accepts these prerequisites, then the concepts of outcome processing and extinction can seamlessly be applied to the control of human behaviour and thinking (Schnider, 2008).

A further important point is that the ability to abandon a currently invalid idea -and thus to synchronize thinking with present reality- does not require full behavioural extinction by repeated exposure. In fact, a currently inappropriate idea or action plan may be entirely appropriate at another moment. What is needed is just the signal 
indicating that an upcoming thought does not relate to current reality. This signal presumably corresponds to the "extinction" signal in reversal learning, which indicates that an anticipated outcome has not occurred. In current "reward system terminology", this would be called the signal of a "negative prediction error".

Animal studies have shown that the main area of brain damage in behaviourally spontaneous confabulation -the posterior medial OFC- is indeed critically involved in behavioural adaptation and outcome processing (Schultz \& Tremblay, 2006). Of particular interest here is an early study by Butter (1969). He found that a lesion of the posterior medial orbitofrontal area in monkeys, corresponding to area 13 , induced a specific failure of extinction. Recent studies confirmed these effects of OFC lesions (Iversen \& Mishkin, 1970; Izquierdo, Suda, \& Murray, 2004). In rats, inactivation of the medial OFC reduced sensitivity to feedback (Dalton, Wang, Phillips, \& Floresco, 2016). Thus, lesions of area 13 deprive an animal of producing the signal necessary for extinction.

Single cell recordings in the OFC of monkeys demonstrated multiple reward-associated responses (Roberts, 2006; Schultz \& Tremblay, 2006). Of particular interest here is the fact that the posterior medial OFC contains neurons that signal the non-occurrence of expected outcomes. Rosenkilde et al. (1981) found neurons, which exclusively fired when a reward was exceptionally not delivered at the expected time. While these neurons were present over the whole OFC, they were particularly concentrated in area 13. Thorpe et al. (1983) obtained similar results. In the context of our discussion, these neurons might actually represent "reality neurons" as they indicate that an anticipated outcome has not occurred. Of note, these neurons apparently only signal the absence of a reward, which was unequivocally expected. No such responses were observed in OFC 
neurons when the non-occurrence of a reward was a possible outcome, that is, in probabilistic reversal learning (Kennerley, Behrens, \& Wallis, 2011).

While these orbitofrontal "reality neurons" briefly fire when an anticipated reward does not occur, neurons in deep dopaminergic structures" (substantia nigra, ventral tegmental area and the striatum) briefly inhibit their firing when such no-events happen (Schultz et al., 1997). They also represent the expected time of reward delivery (Bermudez \& Schultz, 2014). Importantly, reward processing of dopaminergic neurons appears to depend on signals from the orbitofrontal cortex, as shown in rats (Takahashi et al., 2011).

These animal experiments show that the area primarily lesioned in patients with behaviourally spontaneous confabulation contains the neural apparatus to signal that an anticipated outcome has failed to happen. The subcortical dopaminergic structures correspond to those observed in healthy subjects performing a variant of our memory selection task (Treyer et al., 2003).

A basic requirement for this system to synchronize human behaviour and thought with ongoing reality is that it checks any outcome, irrespective of hedonic value. Animal experiments do not allow testing this possibility, as rewards (and punishments) are the main vocabulary of communication with the animals; they tell the animal what response is requested.

We asked healthy human subjects to do a simple task, in which they had to predict behind which one of two coloured rectangles an object was hidden, while their brain activity was measured with $\left[{ }^{15} \mathrm{O}\right] \mathrm{H}_{2} \mathrm{O}$ positron emission tomography (PET) (Schnider, Treyer, \& Buck, 2005). The displayed objects were chosen to have no apparent hedonic value (pencil, button etc.). No reward, not even a comment was provided. In one condition, subjects were told that the object would always appear behind the same 
rectangle, so they should continue to choose the previously correct rectangle. Only occasionally, the object would be absent, indicating that it had switched to the other rectangle. As it turned out, in all conditions in which the subjects had to anticipate the outcome, there was activation of the frontal pole, area 10 , indicating that this area is important for the formulation of a hypothesis. Whenever the outcome was important for the next trial, that is behaviourally relevant, there was activation of area 13 . Thus, the human OFC does process outcomes that have no hedonic value.

Using an adapted version of this experiment with high density EEG, we found that extinction trials, which indicated that the subject had to switch its choice on the next trial, elicited a distinct frontal positivity at about 200-300 milliseconds (Schnider, Mohr, Morand, \& Michel, 2007). A simple unexpected change of the object (for example presentation of a button rather than a pencil) did not elicit this response, underlining the specificity of the trials, which indicated the need to adapt thought and behaviour, that is, extinction trials.

A subsequent study indicated that OFC activity in such a task is indeed driven by behavioural relevance of the outcome rather than the value of reward (Nahum, Gabriel, \& Schnider, 2011). Subjects had to choose the one of two rectangles behind which a game would be hidden. There were three possible outcomes: (1) the game was indeed playing behind this rectangle and reward was delivered; (2) the game was indeed playing behind the chosen rectangle but there was no reward, indicating that the subject should continue to choose this rectangle; (3) there was no reward because the game was not playing behind that rectangle anymore, indicating that the subjects should subsequently switch to the other rectangle. The result was impressive: there was a strong signal, emanating from the medial orbitofrontal cortex, only in extinction trials, that is, when the outcome indicated that behaviour should be adapted on the following 
trial. The simple absence or occurrence of reward did not induce any difference in brain activity.

These studies show that the human OFC also processes outcomes that have no hedonic value. Indeed, the OFC appears to be more interested in the behavioural relevance -the need to adapt behaviour when an anticipation is not valid anymore- than the reward value of outcomes. Interestingly, recent animal studies have also started to consider such an interpretation of OFC function (Kennerley \& Walton, 2011). This capacity predestines the OFC to function as a reality-checking system.

These findings are difficult to compare with other functional imaging studies, which were mainly interested in the OFC's role in learning of reward-associations and decision-making to achieve reward (Kringelbach \& Rolls, 2004; Rushworth, Noonan, Boorman, Walton, \& Behrens, 2011). Localization of OFC activity is questionable: the overwhelming majority of studies used functional magnetic resonance imaging (fMRI), which typically has strong artefacts in the area of the posterior medial orbitofrontal cortex (Stenger, 2006) (in our studies, we used $\left[{ }^{15} \mathrm{O}\right] \mathrm{H}_{2} \mathrm{O}$ PET, which does not have such artefacts). But the fact is that the role of the OFC in adapting thinking and behaviour to ongoing reality, as evident from behaviourally spontaneous confabulation, is simply no topic in neuroscience.

\section{Confabulation and extinction}

The idea that deficient extinction capacity is crucial for the reality confusion characterizing behaviourally spontaneous confabulation and disorientation is derived from clinical observation and compatible with the functions of the posterior medial OFC as described in animal studies and observed in our imaging and electrophysiological studies. But what is the direct evidence of such a link? 
We tested a group of brain-damaged subjects who had amnesia, an orbitofrontal lesion, or both (Nahum et al., 2009). None was in a confusional state and none had degenerative dementia. They made a simple reversal learning task, in which they had to predict which one of two faces displayed on the screen would have a target stimulus (a filled circle or spider) on its nose. They were instructed that the stimulus would normally remain with the same face; if it did not (extinction trials) they should choose the alternate face on the next trial. The result was surprisingly clear: errors during the learning of new associations between a face and the outcome stimulus had no predictive value for the occurrence of behaviourally spontaneous confabulation or disorientation. By contrast, errors after extinction trials, that is, the continued choice of the now incorrect face, was very strongly associated with the degree of disorientation. It also significantly separated behaviourally spontaneous confabulators from the other patients. This result was true for the whole group and for the subgroups of patients matched regarding the severity of amnesia and of patients with orbitofrontal lesions (irrespective of memory performance).

A subsequent study confirmed the strong association of deficient extinction capacity with behaviourally spontaneous confabulation and disorientation (Nahum et al., 2012) but also showed that there was no such association with momentary confabulations or intrusions in a memory test.

\section{A model of orbitofrontal reality filtering}

\section{Insert Figure 1 about here}

Figure 1 displays a model of the influence of reality filtering on the evocation of memories and thoughts. The idea is that thoughts are essentially associations of fragments of memories; the model makes no distinction between them. The timing 
suggested in the model is taken from evoked potential studies of subjects performing

the continuous recognition task (Schnider et al., 2002; Wahlen et al., 2011) and the outcome processing tasks described above (Nahum et al., 2011; Schnider et al., 2007). In this model, the initial step towards an action or an idea (in view of an outcome) lies in the evocation of a memory or associations of memory fragments (a thought), e.g., in reaction to a percept or another thought (Fig. 1A). At 200-300 ms, the OFC verifies the upcoming thought's relation with ongoing reality. If the thought finds a correlate in reality, the filter does not interfere and the thought is processed as "real". If the thought has no correlate in reality, the OFC emits a signal at 200-300 ms, akin to an extinction signal, indicating that the upcoming thought does not relate to the "now", and correspondingly adapts the cortical format of the upcoming thought as "fantasy", that is , a thought which has no correlate in current reality. At 400-600 ms, the thought is recognized and again re-encoded. This sequence -first reality-filtering, then recognition and re-encoding- not only ensures that thought and behaviour are always in synchrony with reality. It also ensures that on the next day, one can distinguish between a real experience and an imagined experience: When they were re-encoded at 400-600 ms, they had different formats.

In reality confusing subjects (Fig. 1B), the evocation of thoughts and memories happens through the same basic processes as in healthy subjects. However, since the orbitofrontal reality-filter is defective, upcoming thoughts are not checked against reality at 200-300 ms, pass as "real", and will be perceived as such -and re-encoded, as far as amnesia allows- at 400-600 ms. This defect explains why some patients may briefly admit that they are actually hospitalized, but then leave the unit a few minutes later in the conviction of having other obligations. The model may also explain the confabulations that patients make about recent doings or their plans. As patients retain 
some of their "unfiltered" fantasies, in explicit memory or through priming, they perceive them subsequently as real experiences and recount them as such (Schnider, Bonvallat, et al., 2005).

This model tries to explain how the brain signals whether an upcoming thought relates to the "now", using the comparison between an anticipation and its correlate in true reality. This mechanism does not, eo ipso, explain why patients would have false ideas about their recent doings, given that it does not "monitor" memories' content or context. However, the model predicts that, as far as patients can store some information thorough explicit learning or priming- including a hint of their own thoughts, these thoughts will be encoded as "real". So patients will conceive of fantasies as if they had really occurred. We tested this prediction in a patient with daily interviews for several days: one minute after intense discussions about earlier family events, she thought that she had actually experienced the discussed event on the same day (Schnider, 2008; Schnider, Bonvallat, et al., 2005).

The cited clinical studies included patients who had acute, mainly focal brain damage (including Wernicke-Korsakoff syndrome). In degenerative dementia, the proposed mechanism does not appear to apply (Joray, Herrmann, Mulligan, \& Schnider, 2004). Reality confusion (disorientation) in dementia is best, albeit weakly, explained by general cognitive decline. It may be that in this case, a sick neocortex may not appropriately react to essentially normal orbitofrontal signals.

\section{Comparison with related theories}

Orbitofrontal reality filtering, as described, only verifies upcoming thoughts' relation with current reality, the "now"; it is not supposed to verify whether an upcoming thought or memory relates to a real event in the past. This is the main conceptual difference between orbitofrontal reality filtering and models postulating mechanisms 
that "monitor" the content (Burgess \& Shallice, 1996; Gilboa et al., 2006; Moscovitch \& Melo, 1997) or the context and source (Johnson, Hashtroudi, \& Lindsay, 1993; Mitchell \& Johnson, 2009) of recalled memories. These capacities rely on different mechanisms than orbitofrontal reality filtering (Bouzerda-Wahlen et al., 2015; Wahlen et al., 2011). Most models have been proposed with no dedicated experiment to test their components or their relevance for a specific kind of confabulation. An exception was Gilboa et al.s (2006) study: They observed that patients described as spontaneous confabulators not only failed in the continuous recognition task that appeared clinically reliable in our studies (Nahum et al., 2012; Schnider \& Ptak, 1999; Schnider, von Däniken, et al., 1996a, 1996b), but also in a task requiring the distinction between items that resembled, but were not identical with, previously seen items. They concluded that diverse failures in the retrieval process, including disruption of different forms of monitoring, might lead up to confabulations. The patients were not matched according to the degree of amnesia, so that the severity of general cognitive failures may have played a more important role than in our studies. We could not reproduce their findings in a group of patients matched regarding the severity of their free recall deficit: under this condition, content monitoring was not predictive for behaviourally spontaneous confabulation or disorientation (Bouzerda-Wahlen et al., 2013). Performance of the two tasks also dissociated electrophysiologically (Wahlen et al., 2011). We found a similar dissociation of reality filtering from source monitoring of visual (Bouzerda-Wahlen et al., 2015) and temporal context (Liverani et al., 2015). Most importantly, while behaviourally spontaneous confabulators typically have difficulty in source attributions (Schnider, Gutbrod, et al., 1996), this defect does not separate them from nonconfabulating amnesics (Johnson, O'Connor, \& Cantor, 1997). Thus mechanisms that verify the content or source and context of memories about the past are -as far as they 
have been experimentally tested- different from orbitofrontal reality filtering, which verifies thoughts' relation with the present.

The idea that confabulations are memories displaced in time, or lacking a "temporal tag", was already formulated by early authors (Talland, 1961; Tiling, 1892; Van der Horst, 1932). This observation refers to all types of confabulations; even intrusions and false recognitions in memory tests may at times be traced back to previous memory tests (Schnider, Gutbrod, et al., 1996). Behaviourally spontaneous confabulators typically fail to place memories in their temporal context (Johnson et al., 1997; Schnider, Gutbrod, Hess, \& Schroth, 1995; Turner, Cipolotti, \& Shallice, 2010), but this failure does not separate them from other amnesics, who also typically fail in such tasks (Hirst \& Volpe, 1982; Huppert \& Piercy, 1976; Johnson et al., 1997; Kopelman, Stanhope, \& Kingsley, 1997). Again, the electrophysiological signature of conscious knowledge about the temporal order of memories differs from orbitofrontal reality filtering (Liverani et al., 2015). The idea of a lost "temporal tag" of memories would not account for a fundamental aspect of behaviourally spontaneous confabulation, namely, that the patients confuse ongoing reality and act according to a false concept of reality. They live in a temporally and spatially displaced reality. In that sense, the notion of disturbed "temporal consciousness", preventing patients from placing themselves correctly in time (Dalla Barba, 2002), comes close to what orbitofrontal reality filtering presumably does. However, in contrast to the latter, disturbed "temporal consciousness" has been held responsible for all types of confabulation, is explored with a confabulation questionnaire, and has been attributed to a dysfunctional, but partially preserved hippocampus (Dalla Barba \& La Corte, 2013). Thus, the concept differs both in terms of applicability and anatomical basis from orbitofrontal reality filtering. 
Diverse authors suspected that confabulations might be rewarding in a situation of personal inferiority induced by the consequences of brain injury (Conway \& Tacci, 1996; Flament, 1957). Fotopoulou et al. (2004) observed that confabulations in response to questions (momentary confabulations) had a positive emotional bias, which might contribute to the production of confabulations. The idea is difficult to translate to the reality confusion characterising behaviourally spontaneous confabulation, as described above. How could the mother's despair to find her baby or the lawyer's constant stress to prepare the imagined session at court -ideas that upset the patients for weeks- be interpreted as "positive experiences"? Of note, a recent evoked potential study did not reveal any influence of positive emotion on orbitofrontal reality filtering, as tested with repeated continued recognition tasks (Liverani, Manuel, Guggisberg, Nahum, \& Schnider, 2016). But clearly, motivation and personality may be critical determinants of whether patients communicate, or not, their false ideas about reality, that is, whether they overtly confabulate or not. Deficient orbitofrontal reality filtering only explains the patients' confusion of reality, not their propensity to talk about it, not event the compulsion to act according on it.

\section{Unknowns and perspectives}

Those who have cared for patients with full-blown behaviourally spontaneous confabulation, similar to the examples given above, cannot but agree that these patients confuse reality. Fully developed behaviourally spontaneous confabulation is a striking model for reality confusion. The paradigms derived from the clinical studies have been useful to explore how the healthy brain adapts thought to ongoing reality.

While extinction capacity has not been specifically tested in amnesic patients by other groups, the continuous recognition tasks with repeated runs has been used (Bajo, Fleminger, Metcalfe, \& Kopelman, 2016; Gilboa et al., 2006; Turner et al., 2010). These 
studies have confirmed failure of "confabulating patients" in the task, but also had false

positives: patients who failed in the task but who were not considered "behaviourally spontaneous". The cause for this discrepancy is not clear. It contradicts our observation that the cessation of acts according to confabulations is accompanied by normalization of performance (Schnider, Ptak, et al., 2000).

Patient classification may be an issue. Indeed, it can be difficult to verify, or exclude, that patients actually act upon their confabulations. Patients with chronic reality confusion may get accustomed to their environment so that their acts may appear entirely appropriate. If one wants to explore the mechanisms of reality confusion and confabulation, it seems advisable to study unequivocally classifiable patients; there is nothing to be gained from labelling any confabulation "(behaviourally) spontaneous". In addition, it is desirable to always document the degree of disorientation, which is a continuous measure of reality confusion (Bouzerda-Wahlen et al., 2013; Nahum et al., 2012; Nahum et al., 2009; Schnider, von Däniken, et al., 1996b), although very severe amnesia with deficient recognition memory may also account for it (Schnider, 2008). The continuous recognition task, as it has been used in most clinical studies (Bajo et al., 2016; Gilboa et al., 2006; Nahum et al., 2012; Schnider, von Däniken, et al., 1996a, 1996b), also requires attention. It is not obvious to make sure that patients' performance failure is really due to an inability to respond to the intended challenges of the task. While healthy subjects normally do it with no difficulty, patients may be rapidly in trouble. Saying "yes" and "no" by pressing a key may be too difficult. In our studies, the experimenter typed the patients' verbal responses in the computer. Patients may forget, or fail to pay attention to, the explicit demands of the second run, which is to detect repetitions within, and only within, the ongoing run. They may say "yes" to any item appearing familiar -or, rarely, avoid false positives by always saying "no" (Ptak \& 
Schnider, 1999). The task used in most clinical studies has the further disadvantage of having more "distracters" (first within-run appearances) than targets. This carries the risk of a spurious increase of false positives in the second run due to a subjective response criterion, that is, of false positives. We remind patients, if necessary, several times throughout the task of what precise question they have to respond to ("It is important that you indicate repetitions of pictures, but only if they have already occurred within this very run.").

In comparison, the reversal learning (extinction) task described above seems straightforward (Nahum et al., 2012; Nahum et al., 2009). No study has yet attempted to replicate our findings.

The model explained in this article postulates that synchronization of thinking and behaviour relies on an interaction of the orbitofrontal cortex, through subcortical connections, with the neocortex. Might this model also apply to other instances of reality confusion, such as, the delusion of schizophrenia and other psychoses? Behaviourally spontaneous confabulation shares with psychosis the conviction about a false reality. Abnormalities in the processing of prediction errors in psychosis, as revealed by fMRI, have been described -although patients' performance was similar to controls (Corlett et al., 2007). A prominent model of psychosis postulates a "hyperdopaminergic state" and an inability to update one's perception of reality in response to errors (Fletcher \& Frith, 2009; Howes \& Kapur, 2009), akin to the model presented here. Indeed, acutely hallucinating schizophrenics have been reported to fail in repeated runs of a continuous recognition task, similar to confabulating patients (Badcock, Waters, Maybery, \& Michie, 2005; Waters, Badcock, Maybery, \& Michie, 2003). The reasons for this failure have yet to be explored. Many view schizophrenia as a disorder of synaptic maturation and brain connectivity rather than, or in addition to, a specific orbitofrontal disease (Bracht et al., 
2014; Dolan, Fletcher, McKenna, Friston, \& Frith, 1999; Honea, Crow, Passingham, \& Mackay, 2005). Orbitofrontal reality filtering, as modelled here, may not only fail when the orbitofrontal cortex is sick, but also, if the neocortex fails to respond to a normal orbitofrontal signal. This situation may explain why disorientation in Alzheimer's disease, a cortical degeneration, is not specifically associated with defective orbitofrontal reality filtering, as measured with the repeated continuous recognition task (Joray et al., 2004). Thus, while the anatomical model of reality adaptation described above (interaction of OFC, subcortical structures, and neocortex) may also apply to psychosis, the underlying defect is presumably different from deficient orbitofrontal reality filtering, proposed to result from the absence of an orbitofrontal "extinction" signal.

Orbitofrontal reality filtering may also play a critical, early role in decision making, the capacity to choose optimally among different options by weighing risks and benefits of a choice and to use outcomes to update the knowledge-base. Decision making heavily involves the orbitofrontal cortex (Rushworth \& Behrens, 2008; Wallis, 2007). But rather than balancing the pros and cons of an impending decision, orbitofrontal reality filtering may be crucial for pre-consciously separating options that currently have a correlate in reality -and may be acted upon- and others that do not.

Two final questions regarding confabulation deserve discussion. Why does only a minority of patients -maybe, 5\% (Schnider, 2008)- with the typical lesions confuse reality and confabulate about their obligations (Schnider, 2008)? And why do behaviourally spontaneous confabulators normally recover from reality confusion, while they remain amnesic (Schnider, Ptak, et al., 2000). What brain area substitutes for the lesioned areas? Current data give some hints: The neurons signalling the absence of a reward at the expected time, which Rosenkilde et al. (1981) found in monkeys, were 
particularly concentrated in, but not limited to the posterior medial OFC. Could it be that the remaining "extinction signalling" cells (which we might call "reality cells") in the intact parts of the OFC compensate for the lost function to a degree that allows thinking (again) to be sufficiently synchronised with reality? Could it even be that the distribution of these cells were genetically determined? There is a hint for genetic variation in reality filtering. We asked healthy subjects to perform an extremely rapid and difficult version of a continuous recognition task to explore modulators of reality filtering. We observed that the inter-individual differences among the test subjects were much more important than the intra-individual modulations induced by dopaminergic medication (Schnider et al., 2010) or tDCS (Manuel et al., 2014). There are rapid and less rapid reality adaptators, presumably reflecting a genetic disposition. This disposition might determine the risk of confusing reality after brain damage or determine the potential of recovery. These questions, however, await specific exploration.

Acknowledgment: The studies reported her were supported by Swiss National Science Foundation, grant no 32003B-155947 .

\section{References}

Alexander, G. E., DeLong, M. R., \& Strick, P. L. (1986). Parallel organization of functionally segregated circuits linking basal ganglia and cortex. Annual Review of Neuroscience, 9, 357-381.

Badcock, J. C., Waters, F. A., Maybery, M. T., \& Michie, P. T. (2005). Auditory hallucinations: failure to inhibit irrelevant memories. Cognitive Neuropsychiatry, $10(2), 125-136$. 
Bajo, A., Fleminger, S., Metcalfe, C., \& Kopelman, M. (2016). Confabulation: What is associated with its rise and fall? A study in brain injury. Cortex (in press).

Bermudez, M. A., \& Schultz, W. (2014). Timing in reward and decision processes. Philosophical Transactions of the Royal Society of London. Series B: Biological Sciences, 369(1637), 20120468. doi: 10.1098/rstb.2012.0468

Bonhoeffer, K. (1901). Die akuten Geisteskrankheiten des Gewohnheitstrinkers. Eine klinische Studie. Jena: Gustav Fischer.

Bouzerda-Wahlen, A., Nahum, L., Liverani, M. C., Guggisberg, A. G., \& Schnider, A. (2015). An electrophysiological dissociation between orbitofrontal reality filtering and context source monitoring. Journal of Cognitive Neuroscience, 27(1), 164-174. doi: 10.1162/jocn_a_00686

Bouzerda-Wahlen, A., Nahum, L., Ptak, R., \& Schnider, A. (2013). Mechanism of disorientation: Reality filtering versus content monitoring. Cortex, 49(10), 26282636.

Bracht, T., Horn, H., Strik, W., Federspiel, A., Razavi, N., Stegmayer, K., . . W Walther, S. (2014). White matter pathway organization of the reward system is related to positive and negative symptoms in schizophrenia. Schizophrenia Research, (in press).

Burgess, P. W., \& Shallice, T. (1996). Confabulation and the control of recollection. Memory, 4, 359-411.

Butter, C. M. (1969). Perseveration in extinction and in discrimination reversal tasks following selective frontal ablations in Macaca mulatta. Physiology and Behavior, 4, 163-171.

Conway, M. A., \& Tacci, P. C. (1996). Motivated confabulation. Neurocase, 2, 325-339. 
Corlett, P. R., Murray, G. K., Honey, G. D., Aitken, M. R., Shanks, D. R., Robbins, T. W., ... Fletcher, P. C. (2007). Disrupted prediction-error signal in psychosis: evidence for an associative account of delusions. Brain, 130(Pt 9), 2387-2400.

Dalla Barba, G., \& La Corte, V. (2013). The hippocampus, a time machine that makes errors. Trends in Cognitive Sciences, 17(3), 102-104.

Dalla Barba, G. F. (2002). Memory, Consciousness and Temporality. Boston: Kluwer Academic Publishers.

Dalton, G. L., Wang, N. Y., Phillips, A. G., \& Floresco, S. B. (2016). Multifaceted Contributions by Different Regions of the Orbitofrontal and Medial Prefrontal Cortex to Probabilistic Reversal Learning. Journal of Neuroscience, 36(6), 19962006. doi: 10.1523/JNEUROSCI.3366-15.2016

Dolan, R. J., Fletcher, P. C., McKenna, P., Friston, K. J., \& Frith, C. D. (1999). Abnormal neural integration related to cognition in schizophrenia. Acta Psychiatrica Scandinavica (Supplementum), 395, 58-67.

Flament, J. (1957). La fabulation dans le syndrome de Korsakov d'étiologie traumatique. Considérations cliniques, psycho-pathologiques et neuro-pathologiques à propos d'une observation de fabulation à caractère mythopathique. Acta Neurologica Belgica, 57, 119-161.

Fletcher, P. C., \& Frith, C. D. (2009). Perceiving is believing: a Bayesian approach to explaining the positive symptoms of schizophrenia. Nature Reviews Neuroscience, $10(1), 48-58$.

Fotopoulou, A., Solms, M., \& Turnbull, O. (2004). Wishful reality distortions in confabulation: a case report. Neuropsychologia, 42(6), 727-744. 
Gilboa, A., Alain, C., Stuss, D. T., Melo, B., Miller, S., \& Moscovitch, M. (2006). Mechanisms of spontaneous confabulations: a strategic retrieval account. Brain, 129(Pt 6), 13991414.

Grabenhorst, F., \& Rolls, E. T. (2011). Value, pleasure and choice in the ventral prefrontal cortex. Trends in Cognitive Sciences, 15(2), 56-67. doi: 10.1016/j.tics.2010.12.004

Hirst, W., \& Volpe, B. T. (1982). Temporal order judgments with amnesia. Brain and Cognition, 1, 294-306.

Honea, R., Crow, T. J., Passingham, D., \& Mackay, C. E. (2005). Regional deficits in brain volume in schizophrenia: a meta-analysis of voxel-based morphometry studies. American Journal of Psychiatry, 162(12), 2233-2245.

Howes, O. D., \& Kapur, S. (2009). The Dopamine Hypothesis of Schizophrenia: Version III--The Final Common Pathway. Schizophrenia Bulletin, 35(3), 549-562.

Huppert, F. A., \& Piercy, M. (1976). Recognition memory in amnesic patients: effect of temporal context and familiarity of material. Cortex, 12, 3-20.

Iversen, S. D., \& Mishkin, M. (1970). Perseverative interference in monkeys following selective lesions of the inferior prefrontal convexity. Experimental Brain Research, $11,376-386$.

Izquierdo, A., Suda, R. K., \& Murray, E. A. (2004). Bilateral orbital prefrontal cortex lesions in rhesus monkeys disrupt choices guided by both reward value and reward contingency. Journal of Neuroscience, 24(34), 7540-7548. doi: 10.1523/JNEUROSCI.1921-04.2004

Johnson, M. K., Hashtroudi, S., \& Lindsay, D. S. (1993). Source monitoring. Psychological Bulletin, 114(1), 3-28.

Johnson, M. K., O'Connor, M., \& Cantor, J. (1997). Confabulation, memory deficits, and frontal dysfunction. Brain and Cognition, 34, 189-206. 
Joray, S., Herrmann, F., Mulligan, R., \& Schnider, A. (2004). Mechanism of disorientation in Alzheimer's disease. European Neurology, 52, 193-197.

Kennerley, S. W., Behrens, T. E., \& Wallis, J. D. (2011). Double dissociation of value computations in orbitofrontal and anterior cingulate neurons. Nature Neuroscience, 14(12), 1581-1589. doi: 10.1038/nn.2961

Kennerley, S. W., \& Walton, M. E. (2011). Decision making and reward in frontal cortex: complementary evidence from neurophysiological and neuropsychological studies. Behavioral Neuroscience, 125(3), 297-317. doi: 10.1037/a0023575

Kopelman, M. D., Stanhope, N., \& Kingsley, D. (1997). Temporal and spatial context memory in patients with focal frontal, temporal lobe, and diencephalic lesions. Neuropsychologia, 35(12), 1533-1545.

Korsakoff, S. S. (1891). Erinnerungstäuschungen (Pseudoreminiscenzen) bei polyneuritischer Psychose. Allgemeine Zeitschrift für Psychiatrie und psychischgerichtliche Medicin, 47, 390-410.

Kringelbach, M. L., \& Rolls, E. T. (2004). The functional neuroanatomy of the human orbitofrontal cortex: evidence from neuroimaging and neuropsychology. Progress in Neurobiology, 72(5), 341-372.

Liverani, M. C., Manuel, A. L., Bouzerda-Wahlen, A., Genetti, M., Guggisberg, A. G., Nahum, L., \& Schnider, A. (2015). Memory in time: Electrophysiological comparison between reality filtering and temporal order judgment. Neuroscience, 289C, 279-288. doi: 10.1016/j.neuroscience.2014.12.064

Liverani, M. C., Manuel, A. L., Guggisberg, A. G., Nahum, L., \& Schnider, A. (2016). No Influence of Positive Emotion on Orbitofrontal Reality Filtering: Relevance for Confabulation. Frontiers in Behavioral Neuroscience, 10, 98. doi: 10.3389/fnbeh.2016.00098 
Manuel, A., David, A., Bikson, M., \& Schnider, A. (2014). Frontal tDCS modulates orbitofrontal reality filtering. Neuroscience, 265C, 21-27.

Mitchell, K. J., \& Johnson, M. K. (2009). Source monitoring 15 years later: what have we learned from fMRI about the neural mechanisms of source memory? Psychological Bulletin, 135(4), 638-677.

Moll, J. (1915). The "amnestic" or "Korsakow's" syndrome with alcoholic etiology: an analysis of thirty cases. The Journal of Mental Science, 61, 424-443.

Moscovitch, M., \& Melo, B. (1997). Strategic retrieval and the frontal lobes: evidence from confabulation and amnesia. Neuropsychologia, 35, 1017-1034.

Nahum, L., Bouzerda-Wahlen, A., Guggisberg, A., Ptak, R., \& Schnider, A. (2012). Forms of confabulation: Dissociations and associations. Neuropsychologia, 50(10), 25242534.

Nahum, L., Gabriel, D., \& Schnider, A. (2011). Human processing of behaviorally relevant and irrelevant absence of expected rewards: a high-resolution ERP study. PLoS One, 6(1, Jan 27), e16173 (16179 pages).

Nahum, L., Ptak, R., Leemann, B., Lalive, P., \& Schnider, A. (2010). Behaviorally spontaneous confabulation in limbic encephalitis: the roles of strategic monitoring and reality filtering. Journal of the International Neuropsychological Society, 16(Nov), 995-1005.

Nahum, L., Ptak, R., Leemann, B., \& Schnider, A. (2009). Disorientation, confabulation, and extinction capacity. Clues on how the brain creates reality. Biological Psychiatry, $65,966-972$

Pavlov, P. I. (1927). Conditioned Reflexes: An Investigation of the Physiological Activity of the Cerebral Cortex. Translated by G. V. Anrep (G. V. Anrep, Trans.). London: Oxford University Press. 
Ptak, R., Birtoli, B., Imboden, H., Hauser, C., Weis, J., \& Schnider, A. (2001). Hypothalamic amnesia with spontaneous confabulations: A clinicopathologic study. Neurology, 56, 1597-1600.

Ptak, R., \& Schnider, A. (1999). Spontaneous confabulations after orbitofrontal damage: The role of temporal context confusion and self-monitoring. Neurocase, 5, 243-250.

Roberts, A. C. (2006). Primate orbitofrontal cortex and adaptive behaviour. Trends in Cognitive Sciences, 10(2), 83-90. doi: 10.1016/j.tics.2005.12.002

Rolls, E. T. (1999). The Brain and Emotion. Oxford: Oxford University Press.

Rosenkilde, C. E., Bauer, R. H., \& Fuster, J. M. (1981). Single cell activity in ventral prefrontal cortex of behaving monkeys. Brain Research, 209, 375-394.

Rushworth, M. F., \& Behrens, T. E. (2008). Choice, uncertainty and value in prefrontal and cingulate cortex. Nature Neuroscience, 11(4), 389-397.

Rushworth, M. F., Noonan, M. P., Boorman, E. D., Walton, M. E., \& Behrens, T. E. (2011). Frontal cortex and reward-guided learning and decision-making. Neuron, 70(6), 1054-1069. doi: 10.1016/j.neuron.2011.05.014

Schnider, A. (2003). Spontaneous confabulation and the adaptation of thought to ongoing reality. Nature Reviews Neuroscience, 4, 662-671.

Schnider, A. (2008). The Confabulating Mind. How the brain creates reality. Oxford: Oxford University Press.

Schnider, A. (2013). Orbitofrontal reality filtering. Frontiers in Behavioral Neuroscience, 7(Jun 10), 67 (doi: 10.3389/fnbeh.2013.00067).

Schnider, A., Bonvallat, J., Emond, H., \& Leemann, B. (2005). Reality confusion in spontaneous confabulation. Neurology, 65(Oct 11), 1117-1119.

Schnider, A., Guggisberg, A., Nahum, L., Gabriel, D., \& Morand, S. (2010). Dopaminergic modulation of rapid reality adaptation in thinking. Neuroscience, 167(3), 583-587. 
Schnider, A., Gutbrod, K., Hess, C. W., \& Schroth, G. (1995). Memory disorder with confabulations after right capsular genu infarction: A frontal lobe syndrome? 155. Tagung der Schweizerischen Neurologischen Gesellschaft, Montreux, 4.-6. Mai 1995. Schweizer Archiv für Neurologie und Psychiatrie, 146, 77 (Abstract).

Schnider, A., Gutbrod, K., Hess, C. W., \& Schroth, G. (1996). Memory without context. Amnesia with confabulations following right capsular genu infarction. Journal of Neurology, Neurosurgery and Psychiatry, 61, 186-193.

Schnider, A., Mohr, C., Morand, S., \& Michel, C. M. (2007). Early cortical response to behaviorally relevant absence of anticipated outcomes: A human event-related potential study. NeuroImage, 35, 1348-1355.

Schnider, A., \& Ptak, R. (1999). Spontaneous confabulators fail to suppress currently irrelevant memory traces. Nature Neuroscience, 2, 677-681.

Schnider, A., Ptak, R., von Däniken, C., \& Remonda, L. (2000). Recovery from spontaneous confabulations parallels recovery of temporal confusion in memory. Neurology, 55, 74-83.

Schnider, A., Treyer, V., \& Buck, A. (2000). Selection of currently relevant memories by the human posterior medial orbitofrontal cortex. Journal of Neuroscience, 20(15), 5880-5884.

Schnider, A., Treyer, V., \& Buck, A. (2005). The human orbitofrontal cortex monitors outcomes even when no reward is at stake. Neuropsychologia, 43, 316-323.

Schnider, A., Valenza, N., Morand, S., \& Michel, C. M. (2002). Early cortical distinction between memories that pertain to ongoing reality and memories that don't. Cerebral Cortex, 12, 54-61.

Schnider, A., von Däniken, C., \& Gutbrod, K. (1996a). The mechanisms of spontaneous and provoked confabulations. Brain, 119, 1365-1375. 
Schnider, A., von Däniken, C., \& Gutbrod, K. (1996b). Disorientation in amnesia. A confusion of memory traces. Brain, 119, 1627-1632.

Schultz, W., Dayan, P., \& Montague, P. R. (1997). A neural substrate of prediction and reward. Science, 275(5306), 1593-1599.

Schultz, W., \& Tremblay, L. (2006). Involvement of primate orbitofrontal neurons in reward, uncertainty, and learning. In D. H. Zald \& S. L. Rauch (Eds.), The orbitofrontal cortex (pp. 173-198). Oxford: Oxford University Press.

Stenger, V. A. (2006). Technical considerations for BOLD fMRI of the orbitofrontal cortex. In D. H. Zald \& S. L. Rauch (Eds.), The orbitofrontal cortex (pp. 423-446). Oxford: Oxford University Press.

Takahashi, Y. K., Roesch, M. R., Wilson, R. C., Toreson, K., O'Donnell, P., Niv, Y., \& Schoenbaum, G. (2011). Expectancy-related changes in firing of dopamine neurons depend on orbitofrontal cortex. Nature Neuroscience, 14(12), 1590-1597. doi: $10.1038 / \mathrm{nn} .2957$

Talland, G. A. (1961). Confabulation in the Wernicke-Korsakoff syndrome. The Journal of Nervous and Mental Diseases, 132, 361-381.

Thorpe, S. J., Rolls, E. T., \& Maddison, S. (1983). The orbitofrontal cortex: neuronal activity in the behaving monkey. Experimental Brain Research, 49, 93-115.

Tiling, T. (1892). Ueber die amnestische Geistesstörung. Allgemeine Zeitschrift für Psychiatrie und psychisch-gerichtliche Medicin, 48, 549-565.

Treyer, V., Buck, A., \& Schnider, A. (2003). Subcortical loop activation during selection of currently relevant memories. Journal of Cognitive Neuroscience, 15, 610-618.

Treyer, V., Buck, A., \& Schnider, A. (2006). Selection of currently relevant words: an auditory verbal memory study using positron emission tomography. Neuroreport, $17,323-327$ 
Turner, M. S., Cipolotti, L., \& Shallice, T. (2010). Spontaneous confabulation, temporal context confusion and reality monitoring: a study of three patients with anterior communicating artery aneurysms. Journal of the International Neuropsychological Society, 16(6), 984-994. doi: 10.1017/S1355617710001104

Van der Horst, L. (1932). Über die Psychologie des Korsakowsyndroms. Monatsschrift für Psychiatrie und Neurologie, 83, 65-84.

Wahlen, A., Nahum, L., Gabriel, D., \& Schnider, A. (2011). Fake or fantasy: Rapid dissociation between strategic content monitoring and reality filtering in human memory. Cerebral Cortex, 21(11), 2589-2598.

Wallis, J. D. (2007). Orbitofrontal cortex and its contribution to decision-making. Annual Review of Neuroscience, 30, 31-56.

Waters, F. A., Badcock, J. C., Maybery, M. T., \& Michie, P. T. (2003). Inhibition in schizophrenia: association with auditory hallucinations. Schizophrenia Research, $62(3), 275-280$.

Wernicke, C. (1900). Grundriss der Psychiatrie in klinischen Vorlesungen. Leipzig: Thieme. 


\section{Legend to Figure}

Figure 1. Functional model of the orbitofrontal reality filter. (a) Normal function: $200 \mathrm{~ms}$ after activation of a memory (thought), reality filtering sets in (Schnider et al., 2002; Wahlen et al., 2011): the upcoming thought is compared to true ongoing reality and an extinciton signal emitted if the upcoming thought (memory) does not relate to ongoing reality (Schnider et al., 2007), limiting the extension of neocortical activation (Schnider, 2003; Wahlen et al., 2011). At 400-600 ms, the activated memory (thought) is recognized and again encoded (Schnider et al., 2002; Wahlen et al., 2011). (b) Hypothetical dysfunction of reality filtering in reality confusing patients: memories (thoughts) are normally activated, but are not checked regarding their relation with reality; all memories are activated, and later recognized and re-encoded, as if they related to ongoing reality. Adapted from Schnider A. (Schnider, 2008), with permission. 
a Activation of a memory

(thought)

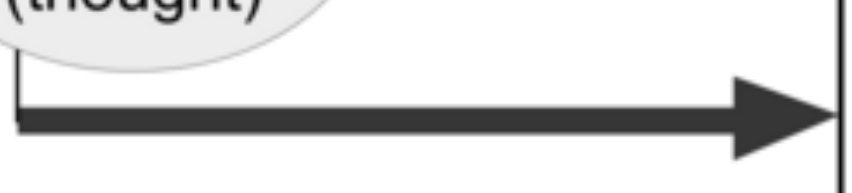

- fantasy

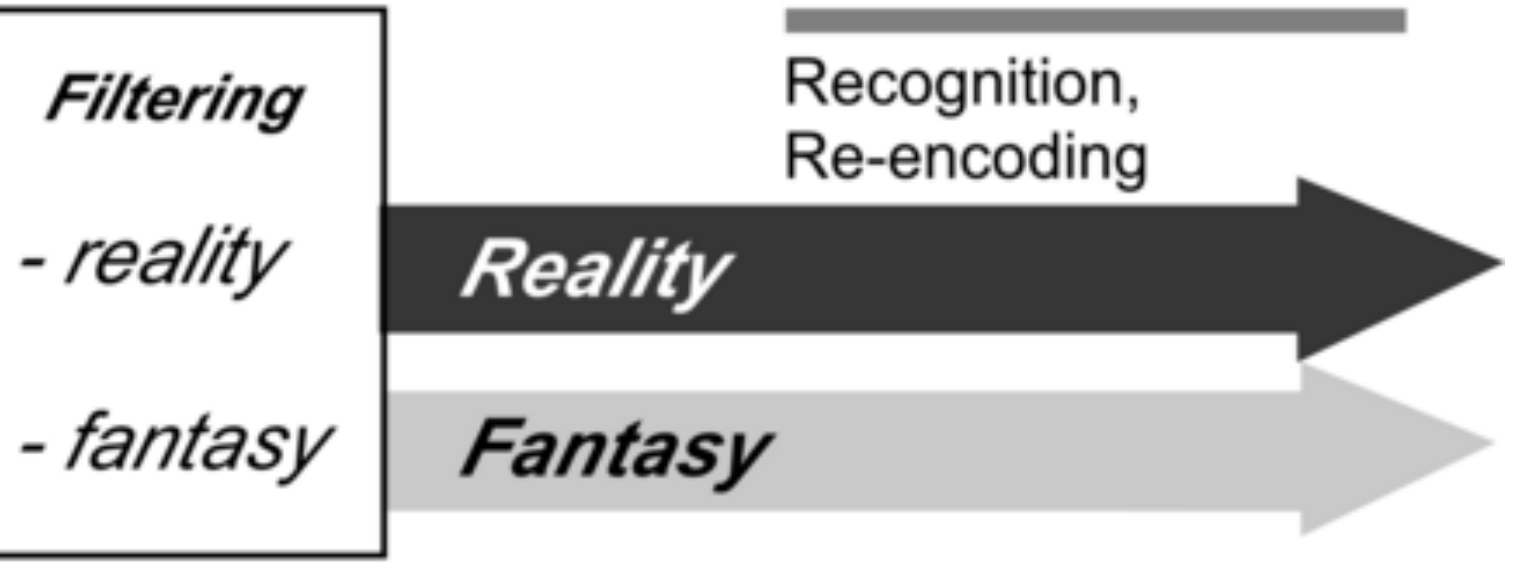

400

500

\begin{tabular}{llcccc}
\hline 100 & 200 & 300 & 500 & 600 \\
& Time after stimulus onset (ms)
\end{tabular}

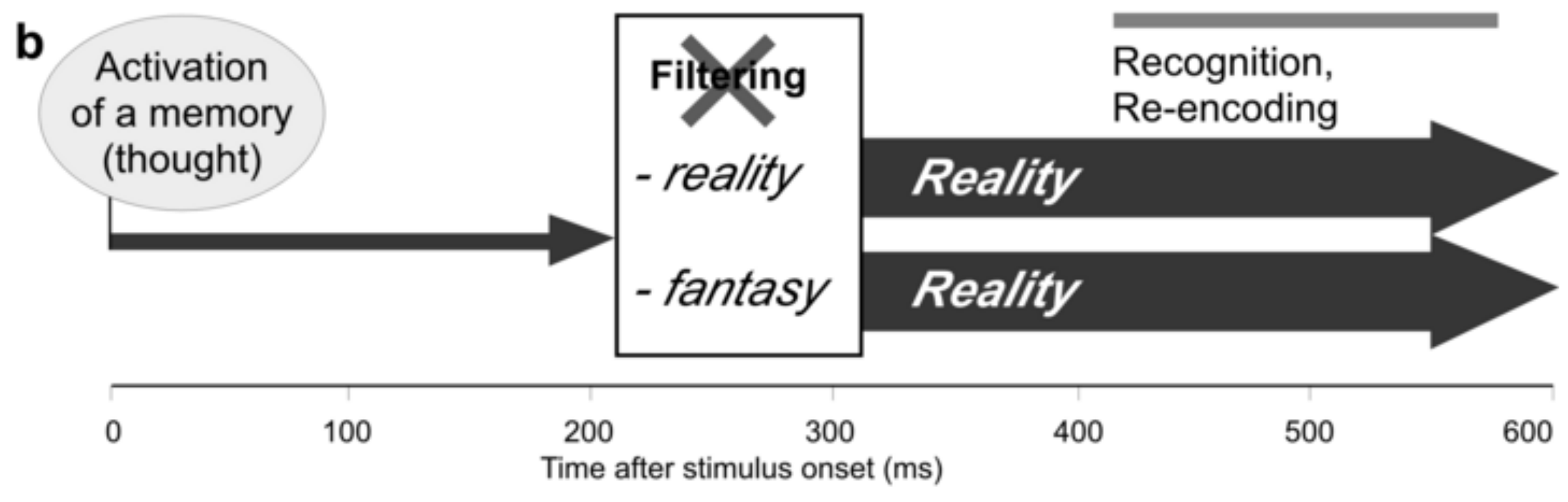

